\title{
Low-Energy He-He Interactions with Phenomenological Potentials
}

\author{
R. F. Bishop \\ Department of Theoretical Physics, University of Manchester, Manchester, U.K., \\ and Daresbury Laboratory, Science Research Council, Daresbury, Warrington, U.K.

\section{H. B. Ghassib*} \\ Department of Physics, University of Jordan, Amman, Jordan \\ and M. R. Strayer \\ Daresbury Laboratory, Science Research Council, Daresbury, Warrington, U.K.
}

(Received August 30, 1976)

The low-energy atom-atom scattering properties are studied for various combinations of He isotopes interacting via a family of semiphenomenological potentials. Some of the potentials bind the $\left({ }^{4} \mathrm{He}\right)_{2}$ molecule and some do not, and it is observed that molecular beam scattering measurements at energies currently accessible cannot resolve the difference between the two types of potentials. Our results are discussed within the framework of possible measurements to resolve this discrepancy. We present a method for solving the Schrödinger equation which is uniformly applicable for both bound and scattering state solutions and which is particularly suited to the types of potentials describing interactions for the rare gas atoms.

\section{INTRODUCTION}

The underlying motivation and interest in understanding the interaction between helium atoms follow in part from the distinct quantal phenomena exhibited by the helium isotopes at low temperatures ${ }^{1}$ and in part from the inherent simplicity of the electronic structure of the helium atom. ${ }^{2}$ The pairwise interaction between helium atomis possesses many of the features displayed by other rare gas atoms. For helium it is the closed 1 s shell that simultaneously accounts for the weakly attractive long-range forces and the strongly repulsive short-range valence forces. These facts, *Supported in part by the University of Jordan. 
coupled with the validity of the Born-Oppenheimer approximation, lead to the representation of the interaction as a static central two-body potential. ${ }^{3}$

Historically our knowledge of the helium interatomic potential has evolved from thermodynamic and steady-state bulk properties. ${ }^{4}$ There currently exists a substantial amount of thermodynamic data on helium in its solid, liquid, and gas phases. However, only a fraction of these data can be meaningfully related to a two-body potential. In particular the absence of a theoretical understanding on a microscopic level of quantum crystals and quantum liquids precludes any direct extraction of atomic potential parameters from the low-temperature solid ${ }^{5}$ and liquid phases. ${ }^{6}$ This is to be contrasted with the dilute gas data ${ }^{7-10}$ and data from the recent precision molecular beam scattering experiments. ${ }^{11-23}$ In this case a complete set of such measurements would in principle suffice to determine a unique energyindependent local potential, ${ }^{24}$ although in practice this aim is not realizable.

The helium potential is best understood in both theory and experiment in either of the limits of small or large separation. Most of the semiphenomenological potentials ${ }^{25-32}$ in present use are piecewise analytic functions of the interatomic separation $r$, which incorporate either or both of the theoretically determined short-range and long-range limits, and which exploit some empirical fit to interpolate between these two limits. The main uncertainty in the potential comes from the range between about 2 and $4 \AA$, which contains the region of the most physical importance, namely the region about the weakly attractive minimum. The long-range part of the interaction is usually given in the form of a multipole expansion, and it is customary to retain only the first two terms, namely the dipole-dipole $\left(C_{6} r^{-6}\right)$ and dipole-quadrupole $\left(C_{8} r^{-8}\right)$ terms. The values of these coefficients have been estimated by many authors ${ }^{33-42}$ and are generally believed to be accurate to $5 \%$ for $C_{6}$ (Ref. 38 ) and $3 \%$ for $C_{8}$ (Ref. 42 ). The omitted terms in the multipole expansion are considered unlikely to contribute more than a few percent to the potential, and then only for larger atomic separations than are relevant to most physical parameters.

Only comparatively recently have high quality $a b$ initio theoretical calculations been reported for the region of the short-range repulsion and near the potential minimum..$^{43-50}$ In general these calculations lead to a simple exponential dependence on the interatomic separations for $r$ between about 1 and $2 \AA$.

In the present work we shall assume that the pairwise interaction between any combination of helium isotopes can be equivalently represented by the same two-body potential. Experimentally there have been data indicating that the interaction is measurably stronger in the ${ }^{3} \mathrm{He}$ system than for ${ }^{4} \mathrm{He}$. Most of the experimental work to date has investigated the ${ }^{4} \mathrm{He}-{ }^{4} \mathrm{He}$ interaction. ${ }^{11-22}$ The analysis of the data, at least within the 
framework of a particular potential model, ${ }^{18}$ suggests that the ${ }^{3} \mathrm{He}-{ }^{3} \mathrm{He}$ interaction is about $3.5 \%$ more attractive than for ${ }^{4} \mathrm{He}-{ }^{4} \mathrm{He}$ in the region of the potential minimum. Other measurements ${ }^{21}$ support this qualitative trend, but find the difference smaller than the experimental error limits.

The current study presents a theoretical comparison of two-body scattering for five of the moderately successful semiphenomenological potentials. The isotopes ${ }^{3} \mathrm{He}$ and ${ }^{4} \mathrm{He}$ are considered, together with the unstable isotope ${ }^{6} \mathrm{He}$. Our basic purpose is to analyze scattering properties of the various model potentials, focusing on whether or not the present data can confirm the existence of a low-energy bound state in the ${ }^{4} \mathrm{He}$ system. In computing the scattering and bound-state properties from the model potentials we present a method of solving the Schrödinger equation which is uniformly applicable for both bound and scattering state solutions, and which is particularly suited to the types of potentials that describe interactions in the rare gas atoms. The method requires the construction of momentum-space matrix elements and is only applicable to potentials that are Fourier-transformable. For bound states, the method displays a high degree of stability and is ideal for computing binding energies of weakly bound systems, while for scattering states, momentum-space matrix techniques provide a systematic method for calculating phase shifts over a wide range of energies.

The five helium model potentials are discussed in Section 2. Some of the potentials fit certain experimental data less well than others, and properties of helium in the gas, liquid, and solid phases as derived from the potentials are compared. The method of solving the bound-state Schrödinger equation and computations of the binding energies of various diatomic nolecules of helium are given in Section 3. The scattering formalism is presented in Section 4 as well as a detailed discussion of the numerical techniques employed. For the ${ }^{3} \mathrm{He}-{ }^{3} \mathrm{He}$ system partial-wave phase shifts are given as a function of energy $(l \leq 7)$ and an effective range expansion is computed for each potential. The computed scattering lengths are accurate to better than $0.1 \%$ and the effective ranges to better than $1.0 \%$. For the ${ }^{4} \mathrm{He}-{ }^{4} \mathrm{He}$ system total elastic and viscosity cross sections are calculated as a function of centerof-mass energy as well as differential cross sections at three different energies. Our results are summarized in Section 5 and are discussed within the framework of possible measurements that will resolve the remaining ambiguities in the interaction.

\section{THE SEMIPHENOMENOLOGICAL POTENTIALS}

In this work the scattering properties of five different $\mathrm{He}-\mathrm{He}$ potentials are compared. In Table I we present a representative cross section of recent 


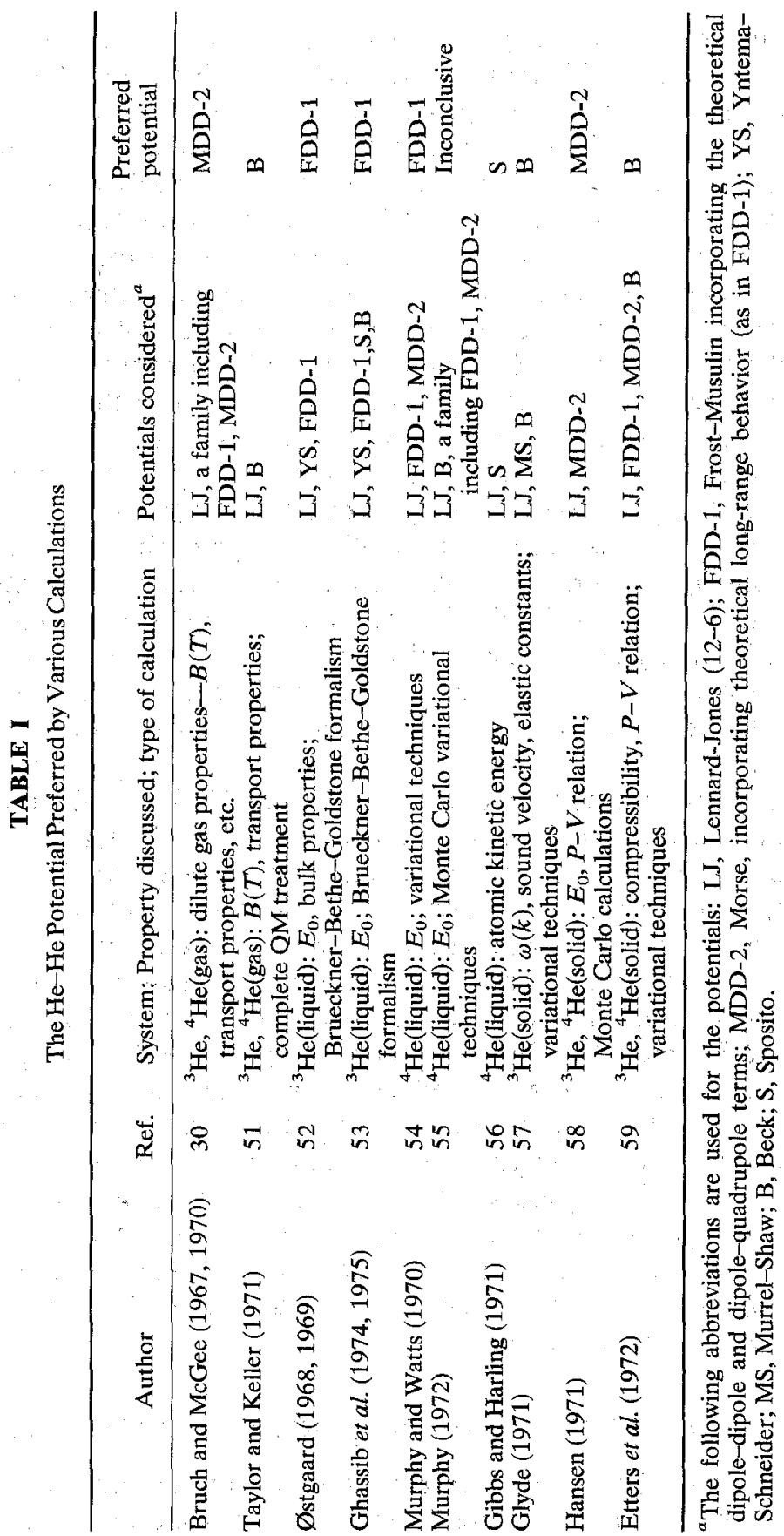


theoretical calculations ${ }^{30,51-59}$ concerned with bulk properties of either or both ${ }^{3} \mathrm{He}$ or ${ }^{4} \mathrm{He}$ in one of the phases gas, liquid, or solid. The last column in Table I indicates the potential that seems to be preferred among those considered. It is seen that the FDD-1, MDD-2, S, and B potentials are widely favored by a range of calculations and it is these potentials, together with one other, that we focus upon. For convenience of classification these interactions may be separated into the following three main classes.

\section{Class A. The Frost-Musulin ${ }^{29}$ Type}

This class comprises the following two potentials:

(i) FDD-1. The Frost-Musulin potential of Bruch and McGee, ${ }^{30}$

$$
V(r)= \begin{cases}-\varepsilon\left[1+c\left(1-r_{m} / r\right)\right] \exp \left[c\left(1-r / r_{m}\right)\right], & r \leq r_{s} \\ -\left(C_{6} r^{-6}+C_{8} r^{-8}\right), & r \geq r_{s}\end{cases}
$$

(ii) MFM. The "modified"* Frost-Musulin ${ }^{29}$ potential, which is given by the short-range $\left(r \leq r_{s}\right)$ part of the FDD-1 potential above, taken for all separations $r$, and which uses the same values of the parameters as FDD-1.

\section{Class B. The Morse Type}

This class also comprises two potentials:

(iii) $M D D-2$. The Morse potential of Bruch and McGee. ${ }^{39}$

(iv) $S$. The Sposito potential. ${ }^{32}$

Both of these potentials are given by the analytic form

$$
V(r)= \begin{cases}-\varepsilon\left\{2 \exp \left[c\left(1-r / r_{m}\right)\right]-\exp \left[2 c\left(1-r / r_{m}\right)\right]\right\}, & r \leq r_{s} \\ -\left(C_{6} r^{-6}+C_{8} r^{-8}\right), & r \geq r_{s}\end{cases}
$$

\section{Class $C$}

This class contains just one potential, namely:

(v) $B$. The Beck potential, ${ }^{31}$ which is given by the analytic form

$$
V(r)=A \exp \left(-\alpha r-\beta r^{6}\right)-B\left(r^{2}+a^{2}\right)^{-3}\left[1+\left(b+3 a^{2}\right) /\left(r^{2}+a^{2}\right)\right]
$$

The values of the various parameters relevant to these potentials are presented in Table II, and the potentials are compared in Fig. 1 in the region of their attractive minima.

Of the five potentials, three (FDD-1, MDD-2, and B) were constructed by their authors along lines similar to those outlined in the introduction. The

\footnotetext{
*The nomenclature "modified" Frost-Musulin potential is perhaps somewhat confusing, since the analytic form is identical to that originally proposed by $F M^{29}$ We have chosen this notation both to avoid confusion with the FDD-1 potential, which is often referred to as the FM potential, and to indicate the particular set of values of the parameters that we have used.
} 
TABLE II

Parameters of the Potentials

\begin{tabular}{|c|c|c|c|c|c|c|c|c|}
\hline Class & Potential & $\begin{array}{l}\varepsilon,{ }^{a} \\
\mathbf{K}\end{array}$ & $r_{m}^{b}$ & $\sigma_{\dot{\dot{A}}}^{c}$ & $c$ & $\begin{array}{l}r_{s}, \\
\AA\end{array}$ & $\begin{array}{l}C_{6}, \\
\mathrm{~K} \AA^{6}\end{array}$ & $\begin{array}{l}C_{8}, \\
\mathrm{~K} \AA^{8}\end{array}$ \\
\hline $\begin{array}{l}\text { A: Frost- } \\
\text { Musulin }\end{array}$ & $\begin{array}{l}\text { FDD }-1^{30} \\
\text { MFM }^{29,30}\end{array}$ & $\begin{array}{l}12.54 \\
12.54\end{array}$ & $\begin{array}{l}2.98 \\
2.98\end{array}$ & $\begin{array}{l}2.649 \\
2.649\end{array}$ & $\begin{array}{l}8.00877 \\
8.00877\end{array}$ & ${ }_{-}^{3.51078}$ & ${ }_{-}^{10213.8}$ & $\underset{d}{27671.4}$ \\
\hline \multirow[t]{2}{*}{ B: Morse } & $\begin{array}{l}\mathrm{MDD}-2^{30} \\
\mathrm{~S}^{32}\end{array}$ & $\begin{array}{r}10.75 \\
9.25\end{array}$ & $\begin{array}{l}3.0238 \\
2.948\end{array}$ & $\begin{array}{l}2.682 \\
2.637\end{array}$ & $\begin{array}{l}6.12777 \\
6.2059\end{array}$ & $\begin{array}{l}3.68280 \\
3.598\end{array}$ & $\begin{array}{r}10213.8 \\
6842.0\end{array}$ & $\begin{array}{l}27671.4 \\
26930.0\end{array}$ \\
\hline & & & & & $\begin{array}{l}A, \quad 1 \\
\mathrm{eV}\end{array}$ & $\AA^{-6} \stackrel{\alpha}{\AA^{-1}}$ & $10^{-4} \stackrel{\beta}{\AA^{-6}}$ & $\begin{array}{ll}a, & b \\
\AA & \AA^{2}\end{array}$ \\
\hline C: Beck & $B^{31}$ & 10.370 & 2.969 & 2.637 & 399.70 .8 & $\begin{array}{ll}69 & 4.390\end{array}$ & $3.746 \quad 0.6$ & $\begin{array}{ll}775 & 2.709\end{array}$ \\
\hline
\end{tabular}

${ }^{a}-\varepsilon$ is the minimum value of the potential.

${ }^{b} r_{m}$ is the separation at the minimum; $V\left(r_{m}\right)=-\varepsilon$.

${ }^{c} \sigma$ is the classical zero-energy turning point; $V(\sigma)=0$.

${ }^{d}$ Not relevant for the MFM potential.

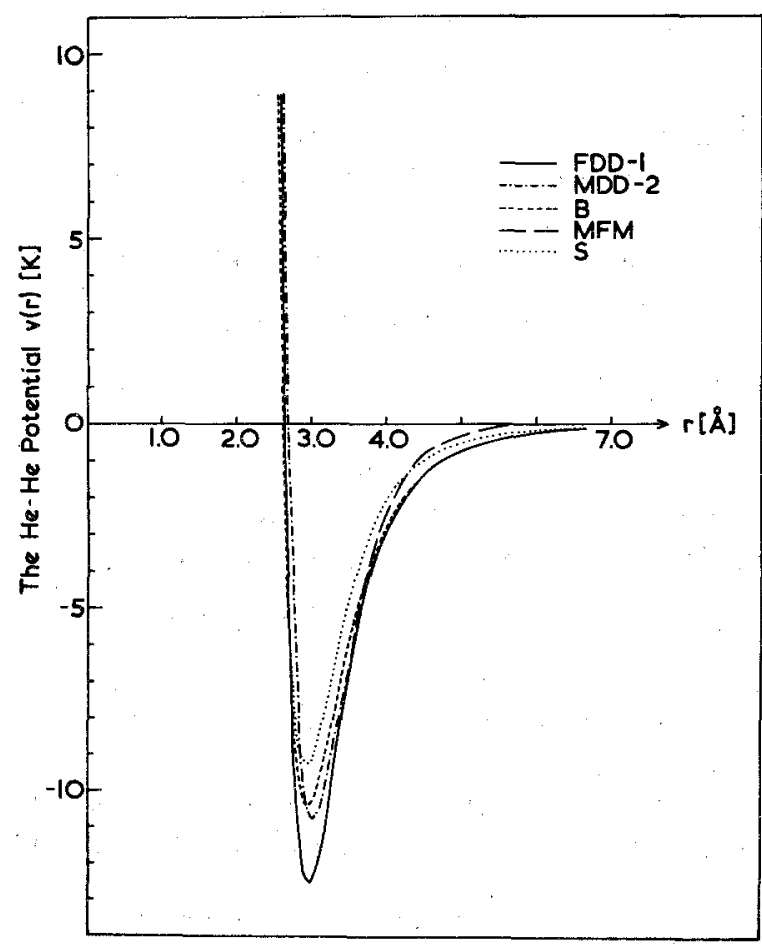

Fig. 1. The five helium interatomic potentials considered in this work, in the region of the potential minimum. 
MFM potential, on the other hand, has the same analytic form as the original Frost-Musulin potential $^{29, *}$ (for all interatomic separations). One might object to its use here on account of its patently incorrect asymptotic behavior at large separations. However, our real motivation for including it is that its Fourier transform has a simple analytic form, which will turn out to provide us with a simple effective check on our numerical computations. Finally, the $S$ interaction was constructed along somewhat different lines. Its short-range part was constructed so as to reproduce the repulsive potential deduced from the extensive high-energy ${ }^{4} \mathrm{He}-{ }^{4} \mathrm{He}$ scattering data of Jordan and Amdur, ${ }^{23}$ whereas its long-range part has the correct asymptotic (dipoledipole and dipole-quadrupole) behavior. Unlike the FDD-1, MDD-2, and $B$ potentials, however, the $S$ potential was adjusted so that it yielded the measured ground-state energy of liquid ${ }^{4} \mathrm{He}$, when combined with the virial theorem, and with the observed radial distribution function $g(r)$ for liquid ${ }^{4} \mathrm{He} .{ }^{60}$ It is therefore not surprising that the resulting potential is somewhat different from the others, which try to reproduce properties of gaseous, rather than liquid, helium. In particular, although its analytic form is identical to that of the MDD-2 potential, the $S$ potential has a considerably shallower minimum at a slightly displaced separation, as seen in Fig. 1. This has evidently resulted from the effects of the many-body medium of the surrounding liquid, and is in general agreement with theoretical calculations on two-body potentials for nonpolar liquids. ${ }^{61}$ Although the $\mathrm{S}$ potential might represent an excellent potential for liquid ${ }^{4} \mathrm{He}$, it is by no means clear whether it is a good representation for use in liquid ${ }^{3} \mathrm{He}$, or between other isotopic pairs.

It is important to point out that, notwithstanding these considerations for the $\mathrm{S}$ potential, and the discussion of the ${ }^{3} \mathrm{He}-{ }^{3} \mathrm{He}$ and ${ }^{4} \mathrm{He}-{ }^{4} \mathrm{He}$ potential differences mentioned in the previous section, we shall use each of these five potentials as representing the interaction between any pair of helium isotopes. Apart from the common isotopes ${ }^{3} \mathrm{He}$ and ${ }^{4} \mathrm{He}$, we consider also the isotope ${ }^{6} \mathrm{He}$. The isotope ${ }^{6} \mathrm{He}$ is unstable and may be produced in such nuclear reaction as ${ }^{9} \mathrm{Be}(\mathrm{n}, \alpha)^{6} \mathrm{He}$, and since it has a comparatively long half-life of $0.802 \mathrm{sec}$, it may prove possible to perform atomic beam experiments with ${ }^{6} \mathrm{He}$ projectiles. Most of our results are sensitive to the precise values of the atomic masses, and these are listed in Table III together with values of the other physical constants used. We note that the basic conversion unit implied by these values $\dagger$ is

$$
\hbar^{2} / m_{3}=16.0838 \mathrm{~K} \AA^{2}
$$

* See preceding footnote.

TThe incorrect value $\hbar^{2} / m_{3}=16.36 \mathrm{~K} \AA^{2}$ seems to be widely used, and appears to have propagated through the liquid helium literature from the pioneering work of Brueckner and Gammel. $^{62}$ 
TABLE III

Physical Constants

\begin{tabular}{|c|c|}
\hline Isotope & Atomic mass, ${ }^{70}$ amu $\left({ }^{12} \mathrm{C}\right)$ \\
\hline $\begin{array}{l}{ }^{3} \mathrm{He} \\
{ }^{4} \mathrm{He} \\
{ }^{6} \mathrm{He}\end{array}$ & $\begin{array}{l}m_{3}=3.016030 \\
m_{4}=4.002603 \\
m_{6}=6.01889\end{array}$ \\
\hline \multicolumn{2}{|c|}{$\begin{array}{l}1 \mathrm{amu}\left({ }^{12} \mathrm{C}\right)=9.31502 \times 10^{8} \mathrm{eV} \\
k_{\mathrm{B}}=8.61735 \times 10^{-5} \mathrm{eV} \mathrm{K}^{-1} \\
\hbar c=1973.29 \mathrm{eV} \AA\end{array}$} \\
\hline
\end{tabular}

As a final note, it is important to realize that each of the five potentials documented above possesses a Fourier transform. This will turn out to be important since the methods that we later adopt are only applicable to Fourier-transformable potentials. Their use would be precluded for such interactions as those of Lennard-Jones ${ }^{4}$ or Yntema and Schneider, ${ }^{25}$ which are too singular near the origin. The theoretical predictions for the asymptotic behavior of the interatomic potential at short and large separations, however, strongly support the idea that a realistic potential should possess a Fourier transform.

\section{MOLECULES OF THE HELIUM ISOTOPES}

In this section we discuss the binding energies of the possible diatoms formed from two helium atoms, each of which can have mass number $A=3$, 4 , or 6. It turns out that none of the five potentials discussed above is sufficiently attractive to bind either $\left({ }^{3} \mathrm{He}\right)_{2}$ or ${ }^{3} \mathrm{He}-{ }^{4} \mathrm{He}$. The only other possible diatom formed from the stable isotopes, $\left({ }^{4} \mathrm{He}\right)_{2}$, is either unbound or bound with a very small energy, depending on the potential considered. For this reason, the unstable isotope ${ }^{6} \mathrm{He}$ is also considered and we present results for the binding energies of the molecules $\left({ }^{4} \mathrm{He}\right)_{2},{ }^{4} \mathrm{He}-{ }^{6} \mathrm{He}$, and $\left({ }^{6} \mathrm{He}\right)_{2}$.

For two atoms of mass $m_{i}$ and $m_{j}$, with reduced mass $\mu_{i j}$, the Schrödinger equation in the bound-state region $(E<0)$ is

$$
\left[-\left(\hbar^{2} / 2 \mu_{i j}\right) \nabla^{2}+V(\mathbf{x})\right] \psi_{i j}(\mathbf{x})=E \psi_{i j}(\mathbf{x})
$$

In order to achieve high accuracy, among other reasons, we have chosen to solve for the possible bound states in the momentum representation.

*The possibility ${ }^{3} \mathrm{He}-{ }^{6} \mathrm{He}$ is not considered separately since the reduced mass for this diatom is very nearly equal to $\frac{1}{2} m_{4}$ and therefore it will have a binding energy almost identical to that of $\left({ }^{4} \mathrm{He}\right)_{2}$. 
Converting Eq. (1) and taking the partial wave decomposition, we obtain

$$
\left(p^{2}-\frac{2 \mu_{i j} E}{\hbar^{2}}\right) \psi_{i j}^{(l)}(p)=-\int_{0}^{\infty} \frac{q^{2} d q}{2 \pi^{2}} \frac{2 \mu_{i j}}{\hbar^{2}} V_{l}(p, q) \psi_{i j}^{(l)}(q)
$$

where

$$
V_{l}(p, q)=4 \pi \int_{0}^{\infty} d x x^{2} j_{l}(p x) V(x) j_{l}(q x)
$$

Equation (2) is a homogeneous integral equation for the possible eigenstates, the kernel of which can be symmetrized by the substitution

$$
\psi_{i j}^{(l)}(p)=\tilde{g}_{l}(p) p^{-1}\left(p^{2}+\frac{2 \mu_{i j}|E|}{\hbar^{2}}\right)^{-1 / 2}
$$

Writing

$$
2 \mu_{i j} \hbar^{-2}|E| \equiv \varepsilon
$$

we find

$$
\tilde{g}_{l}(p)=\left(-\frac{2 \mu_{i j}}{m_{3}}\right) \int_{0}^{\infty} d q \frac{p q}{2 \pi^{2}} \frac{m_{3}}{\hbar^{2}} V_{l}(p, q)\left[\left(p^{2}+\varepsilon\right)\left(q^{2}+\varepsilon\right)\right]^{-1 / 2} \tilde{g}_{l}(q)
$$

The energy eigenvalue $\varepsilon$ now enters. Eq. (5) in a rather complicated nonlinear way and it is useful to regard $\varepsilon$ as a parameter and to treat the overall strength of the potential as the eigenvalue. That is, we solve the linear eigenvalue problem

$$
\int_{0}^{\infty} d q \frac{p q}{2 \pi^{2}} \frac{m_{3}}{\hbar^{2}} V_{l}(p, q)\left[\left(p^{2}+\varepsilon\right)\left(q^{2}+\varepsilon\right)\right]^{-1 / 2} \tilde{g}_{l}(q)=\lambda \tilde{g}_{l}(p)
$$

for the eigenvalue $\lambda$ as a function of the parameter $\varepsilon$. For a given pair $(i, j)$, the binding energy is obtained using Eq. (4), from the value of $\varepsilon$ appropriate to the eigenvalue $\lambda(\varepsilon)=\lambda_{i j} \equiv-m_{3} / 2 \mu_{i j}$. The parameter $\lambda^{-1}$ thus plays the role of an overall variable strength factor for the potential. In general Eq. (6) will have an infinite set of eigenvalues in $\lambda$ for a given $\varepsilon$, which are determined by the strengths of the given potential that sustain a bound state of energy $E=-\left(\hbar^{2} / 2 \mu_{i j}\right) \varepsilon$. Quite clearly, for the potentials of the form considered here (and see Fig. 1), the spectrum of eigenvalues $\lambda$ spans both positive and negative values. For the problem at hand the only relevant eigenvalue is the lowest, namely the negative eigenvalue of greatest absolute magnitude.

The eigenvalue equation (6) may now be solved by any of the standard numerical techniques. We have chosen to use matrix diagonalization after 
the integral has been discretized by an appropriate choice of a numerical quadrature rule over the interval $(0, \infty)$, using a set of integration points $q_{\alpha}$ and corresponding weights $w_{\alpha} ; \alpha=1,2, \ldots, N$. In the present work Gaussian integration has been used and found to provide high accuracy for a relatively small number $N$ of mesh points. The Gaussian integration points $x_{\alpha}$ provided on the interval $(-1,1)$ have been transformed onto the points $q_{\alpha}$ by use of the mapping

$$
q \propto \tan \left[\frac{1}{4} \pi(1+x)\right]
$$

The substitution into Eq. (6) of the transformation

$$
g(p)=[w(p)]^{-1 / 2} \tilde{g}_{l}(p)
$$

then ensures that the resulting finite-dimensional matrix eigenvalue equation

$$
K_{\alpha \beta} g_{\beta}=\lambda g_{\alpha}, \quad g_{\alpha} \equiv g\left(p_{\alpha}\right)
$$

has a symmetric kernel $K_{\alpha \beta}$ given by

$$
K_{\alpha \beta}=\left[\frac{w_{\alpha} w_{\beta}}{\left(p_{\alpha}^{2}+\varepsilon\right)\left(p_{\beta}^{2}+\varepsilon\right)}\right]^{1 / 2} \frac{p_{\alpha} p_{\beta}}{2 \pi^{2}} \frac{m_{3}}{\hbar^{2}} V_{l}\left(p_{\alpha}, p_{\beta}\right)
$$

Equations (7) and (8) have been solved in the $S$-wave $(l=0)$ channel for each of the five potentials under consideration, giving the set of curves $\lambda=\lambda(\varepsilon)$ shown in Fig. 2. None of the five potentials is sufficiently strong to bind any of the possible $\mathrm{He}_{2}$ molecules in a state of nonzero relative angular momentum $(l \geq 1)$. The number of Gaussian integration points $N$ has been varied between 24 and 192 in order to examine the convergence of the quadrature approximation. In every case $N=96$ was found to give values of the eigenvalue $\lambda(\varepsilon)$ accurate to better than $10^{-2} \%$. In all of the numerical work reported we have actually employed $N \geq 96$.

The binding energies (if a bound state exists) of the molecules $\left({ }^{4} \mathrm{He}\right)_{2}$, ${ }^{4} \mathrm{He}-{ }^{6} \mathrm{He}$, and $\left({ }^{6} \mathrm{He}\right)_{2}$ can be read off from Fig. 2 from the respective eigenvalues $\lambda \equiv-m_{3} / 2 \mu$ equal to $-0.75352,-0.62731$, and -0.50109 . Accurate values are listed in Table IV, where they are compared with values quoted by Bruch and $\mathrm{McGee}{ }^{30}$ and Beck. ${ }^{63}$

Our results indicate that if a bound state exists at all for the $\left({ }^{4} \mathrm{He}\right)_{2}$ system, it will be extremely loosely bound. This is in agreement with the theoretical estimates of Pais and Uhlenbeck ${ }^{64}$ and with earlier numerical estimates using various $\mathrm{He}-\mathrm{He}$ potentials and computational techniques. ${ }^{65,66}$ 


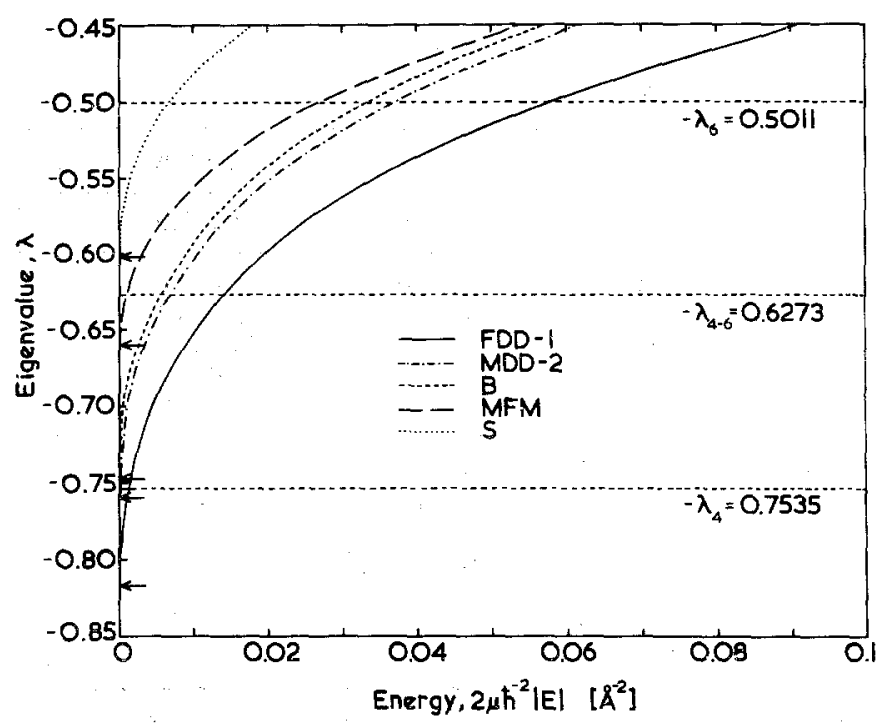

Fig. 2. The lowest eigenvalue $\lambda$ of Eqs. (7) and (8) in the $l=0$ channel, as a function of the reduced energy $2 \mu \hbar^{-2}|E|$, for the five potentials considered. The arrows indicate the end points of each curve at $E=0$. The intercepts, if any, with the lines $\lambda=\lambda_{6}=-0.5011, \lambda=\lambda_{4-6}=$ -0.6273 , and $\lambda=\lambda_{4}=-0.7535$ give, respectively, the binding energies of the molecules $\left({ }^{6} \mathrm{He}\right)_{2},{ }^{4} \mathrm{He}-{ }^{6} \mathrm{He}$, and $\left({ }^{4} \mathrm{He}\right)_{2}$.

\section{HELIUM-HELIUM SCATTERING PHENOMENA}

We now wish to compare the predictions obtained from the various interatomic potentials for scattering processes. Any two-body scattering phenomenon can be described in its complete off-shell generality by the transition amplitude or $t$-matrix operator. Scattering of particles $i$ and $j$ from initial states of momentum $\hbar \mathbf{p}_{i}$ and $\hbar \mathbf{p}_{j}$ to final states $\hbar \mathbf{p}_{i}^{\prime}$ and $\hbar \mathbf{p}_{j}^{\prime}$ with a total (conserved) energy $E$ is described by a $t$-matrix, written in the two-body momentum representation as $t\left(\mathbf{p}_{i}, \mathbf{p}_{i} ; \mathbf{p}_{i}^{\prime}, \mathbf{p}_{j}^{\prime} ; E\right)$. In general the inequalities

$$
\frac{p_{i}^{2}}{2 m_{i}}+\frac{p_{j}^{2}}{2 m_{j}} \neq \frac{E}{\hbar^{2}} \neq \frac{p_{i}^{\prime 2}}{2 m_{i}}+\frac{p_{j}^{\prime 2}}{2 m_{j}}
$$

hold and define what is meant by off-shell scattering. The transition is best described in the CM frame of the scattering pair in terms of the relative and total momentum variables, respectively,

$$
\begin{aligned}
& \mathbf{p}=\left(m_{j} \mathbf{p}_{i}-m_{i} \mathbf{p}_{j}\right) /\left(m_{i}+m_{i}\right) \\
& \mathbf{p}^{\prime}=\left(m_{j} \mathbf{p}_{i}^{\prime}-m_{i} \mathbf{p}_{j}^{\prime}\right) /\left(m_{i}+m_{j}\right) \\
& \mathbf{P}=\mathbf{p}_{i}+\mathbf{p}_{j}=\mathbf{p}_{i}^{\prime}+\mathbf{p}_{j}^{\prime}
\end{aligned}
$$


TABLE IV

Bound States of the Molecules of Helium Isotopes

\begin{tabular}{|c|c|c|c|c|}
\hline \multirow[b]{2}{*}{ Author } & \multirow[b]{2}{*}{ Potential } & \multicolumn{3}{|c|}{ Binding energy, ${ }^{\alpha} \mathrm{K}$} \\
\hline & & $\left({ }^{4} \mathrm{He}\right)_{2}$ & ${ }^{6} \mathrm{He}-{ }^{4} \mathrm{He}$ & $\left({ }^{6} \mathrm{He}\right)_{2}$ \\
\hline Present work & $\begin{array}{l}\text { FDD-1 } \\
\text { MDD-2 } \\
\text { B } \\
\text { MFM } \\
\text { S }\end{array}$ & $\begin{array}{c}0.0140(1) \\
0.000135(3) \\
-_{b} \\
-_{-}{ }^{b}\end{array}$ & $\begin{array}{c}0.1418(1) \\
0.0664(1) \\
0.0554(1) \\
0.00890(2) \\
\quad \_b\end{array}$ & $\begin{array}{l}0.4558(2) \\
0.2950(1) \\
0.2669(1) \\
0.2181(1) \\
0.0533(1)\end{array}$ \\
\hline Bruch and $\mathrm{McGee}{ }^{30}$ & $\begin{array}{l}\text { FDD-1 } \\
\text { MDD-2 }\end{array}$ & $\begin{array}{c}0.0137 \\
{[0.0109]^{c}} \\
0.000134 \\
{[0.000133]^{c}}\end{array}$ & $-^{d}$ & $-^{d}$ \\
\hline Beck $^{31}$ & $\begin{array}{l}\text { FDD-1 } \\
\text { B }\end{array}$ & $\begin{array}{r}0.0067 \\
-\end{array}$ & $\begin{array}{l}0.1171 \\
0.0431\end{array}$ & $\begin{array}{l}0.4145 \\
0.2405\end{array}$ \\
\hline
\end{tabular}

${ }^{a}$ The figure in parentheses after the entry indicates the estimated error to the last significant figure quoted.

${ }^{b}$ No bound state.

${ }^{c}$ These authors quote two values for the binding energy: The first value is obtained from an "exact" solution of the radial Schrödinger equation; the value in brackets is an estimate based on the $S$-wave scattering length.

${ }^{d}$ Not quoted by these authors.

where momentum conservation is guaranteed. The $t$-matrix may now be specified by matrix elements $t\left(\mathbf{p}, \mathbf{p}^{\prime} ; s\right)$ in this frame, where the variable $s$ is proportional to the available energy $\varepsilon$ in the $\mathrm{CM}$ frame,

$$
s=2 \mu_{i j} \hbar^{-2} \cdot \varepsilon=2 \mu_{i j} \hbar^{-2}\left[E-\frac{1}{2} \hbar^{2} \mathbf{P}^{2} /\left(m_{i}+m_{j}\right)\right]
$$

and where $\mu_{i j}$ is the reduced mass of particles $i$ and $j$. The variables $E$ and $\mathbf{P}$ naturally enter the scattering description only in the combination specified by the parameter $s$. The Schrödinger equation that describes the general two-body scattering process above may be rewritten as the LippmannSchwinger equation for the $t$-matrix, which after an angular momentum decomposition may be written as

$$
t_{l}\left(p, p^{\prime} ; s\right)=\frac{2 \mu_{i j}}{\hbar^{2}} V_{l}\left(p, p^{\prime}\right)-\int_{0}^{\infty} \frac{d q q^{2}}{2 \pi^{2}} \frac{2 \mu_{i j}}{\hbar^{2}} \frac{V_{l}(p, q) t_{l}\left(q, p^{\prime} ; s\right)}{q^{2}-s-i \eta}
$$

where $\eta$ is a positive infinitesimal that acts as a stylized reminder of the outgoing wave boundary condition that is built into the definition of the $t$-matrix. With our choice of normalization, the fully on-shell $t$-matrix is 
related to the phase shifts $\delta_{l}$ by the relation

$$
t_{l}\left(p, p ; p^{2}\right)=-4 \pi p^{-1} e^{i \delta_{l}(p)} \sin \delta_{l}(p)
$$

In the scattering region $(s>0)$ the kernel of the integral equation (9) becomes singular at the point $q=+s^{1 / 2}$, and it is necessary that this singularity should be removed from the equation before a numerical solution is attempted. Due to the in term in Eq. (9), the $t$-matrix is complex imaginary for $s>0$ and for many purposes it is more convenient to deal with the analogous $k$-matrix, which has explicitly real momentum-space matrix elements, and which is obtained as the solution to an integral equation identical to Eq. (9) except that $\eta$ is set to zero and the resulting integral is regarded as of principal value type.

The $k$-matrix is thus the solution to a completely identical Schrödinger equation, but with the outgoing-wave boundary condition replaced by a standing-wave boundary condition. From a knowledge of either the $t$-matrix or the $k$-matrix, the other is readily obtained by use of two-body unitarity. In particular, the fully on-shell $k$-matrix is given by

$$
k_{l}\left(p, p ; p^{2}\right)=-4 \pi p^{-1} \tan \delta_{l}(p)
$$

From numerical solutions, the $k$-matrix counterpart of Eq. (9) has a term (which is identically zero) subtracted from the integrand, which has the effect of replacing the principal value condition by a smooth, nonsingular integrand. The resulting integral equation,

$$
\begin{aligned}
& k_{l}\left(p, p^{\prime} ; s\right)= \frac{2 \mu_{i j}}{\hbar^{2}} V_{l}\left(p, p^{\prime}\right) \\
&-\frac{2 \mu_{i j}}{\hbar^{2}} \int_{0}^{\infty} \frac{d q}{2 \pi^{2}} \frac{q^{2} V_{l}(p, q) k_{l}\left(q, p^{\prime} ; s\right)-s V_{l}(p, \kappa) k_{l}\left(\kappa, p^{\prime} ; s\right)}{q^{2}-s} \\
& \kappa \equiv+s^{1 / 2}
\end{aligned}
$$

is now discretized exactly as in Section 3 by use of an appropriate $N$-point quadrature rule. The point $q=\kappa$ has now to be added to the $N$ integration points, due to the smoothing procedure adopted, and the resulting set of $N+1$ linear equations is solved by standard matrix inversion methods. In particular, the phase shifts $\delta_{l}(p)$ are readily obtained by use of Eq. (11). In all of the numerical work reported here, Gaussian quadrature has been employed with the same tangent mapping as described in Section 3. The convergence of the discretization procedure has been tested by varying the number $N$ of integration points, and in all cases $N=96$ was found to give extremely accurate results. 
As an indication of the type of results obtained, we show in Fig. 3 calculations for ${ }^{3} \mathrm{He}-{ }^{3} \mathrm{He}$ elastic scattering, parametrized in terms of phase. shifts, of which we display the first seven obtained with the FDD-1 potential. Each of these phase shifts tends asymptotically to zero as the relative momentum approaches infinity, and the fact that they do so only very slowly is a clear reflection of the strong short-range repulsion. It is this facet of the potentials that, when coupled with their large width, makes numerical computations with them so difficult. The dependence of our results on the potential is illustrated in Fig. 4 for the $S$-wave phase shift for ${ }^{3} \mathrm{He}-{ }^{3} \mathrm{He}$ scattering. The fact that each of the phase shifts tends to zero at zero relative angular momentum is related by Levinson's theorem ${ }^{67}$ to the fact that none of the potentials is attractive enough to bind the $\left({ }^{3} \mathrm{He}\right)_{2}$ molecule, as already discussed in the previous section.

Low-energy elastic scattering data are best parametrized in terms of the $S$-wave scattering length $a_{0}$ and effective range $r_{0}$ defined by the asymptotic

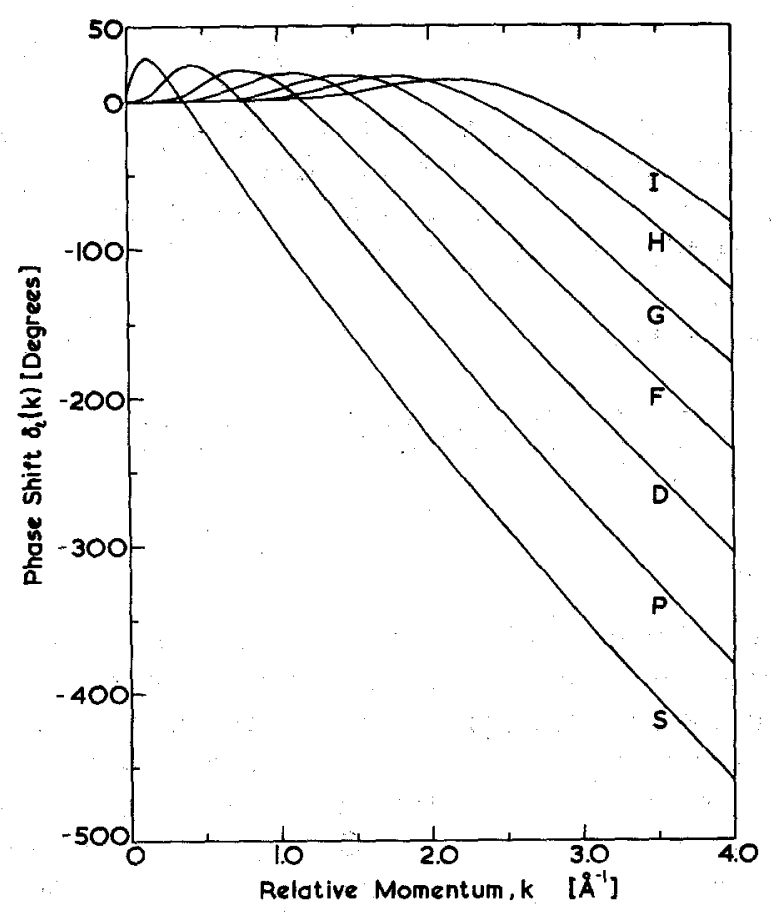

Fig. 3. The first seven phase shifts $\delta_{l}(l=0,1, \ldots, 6)$ as a function of relative momentum $k$ for ${ }^{3} \mathrm{He}-{ }^{3} \mathrm{He}$ scattering via the FDD-1 potential. 


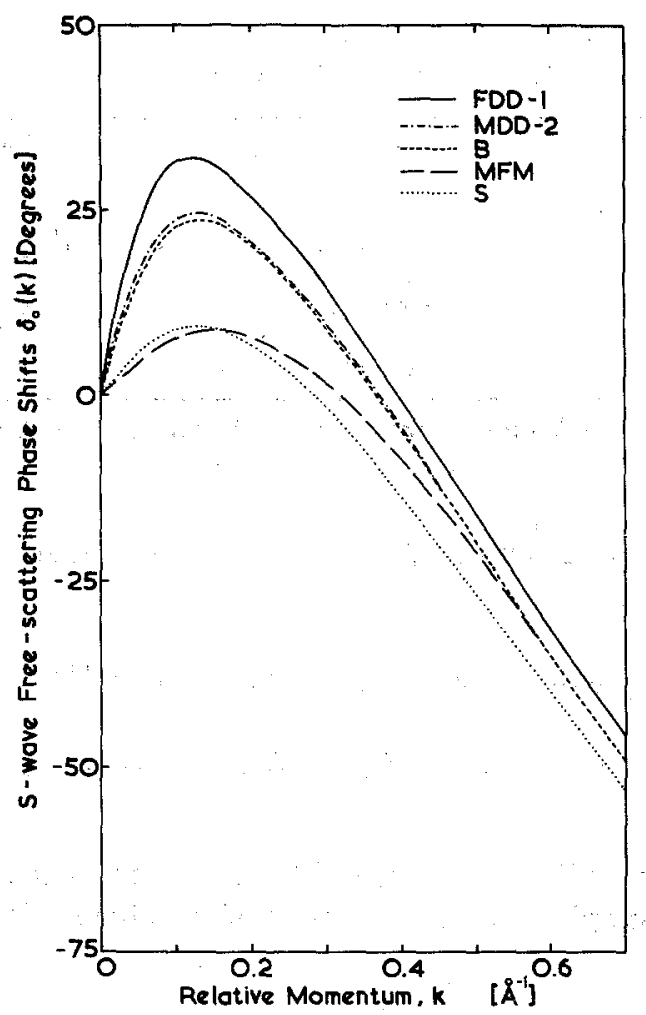

Fig. 4. A comparison of the ${ }^{3} \mathrm{He}-{ }^{3} \mathrm{He} S$-wave phase shifts $\delta_{0}$ as functions of relative momentum $k$ for the five interactions considered.

expansion of the $S$-wave phase shift,

$$
k \cot \delta_{0}(k) \underset{k \rightarrow 0}{\longrightarrow}-a_{0}^{-1}+\frac{1}{2} r_{0} k^{2}+O\left(k^{4}\right)
$$

Our results for these parameters (for ${ }^{3} \mathrm{He}-{ }^{3} \mathrm{He}$ scattering) with the potentials considered are shown in Table $\mathrm{V}$.

The elastic scattering of ${ }^{4} \mathrm{He}-{ }^{4} \mathrm{He}$ is the system that has been most studied experimentally. Most of the data to date have been obtained for the total cross section $\sigma_{T}(k)$. Due to the requirement of Bose statistics the differential cross section is given by*

$$
\frac{d \sigma(k)}{d \Omega}=\frac{1}{2}|f(\theta)+f(\pi-\theta)|^{2}
$$

* Schiff ${ }^{68}$ gives the physically measurable differential cross section as twice that expressed by Eq. (14). The factor $\frac{1}{2}$ in Eq. (14) arises from the usual factor $2^{-1 / 2}$ in the normalized symmetric wave function, and this result should be multiplied by an additional factor of two to account for the fact that the two particles are indistinguishable. We have adopted the definition of Eq. (14) to comply with the majority of the experimental papers in this field. 
TABLE V

The $S$-Wave ${ }^{3} \mathrm{He}^{-}{ }^{3} \mathrm{He}$ Scattering Parameters for Various Potentials ${ }^{a}$

\begin{tabular}{lcc}
\hline Potential & Scattering length $a_{0}$, & $\begin{array}{c}\text { Effective range } r_{0}, \\
\AA\end{array}$ \\
\hline FDD-1 & $\AA$ & $11.7(1)$ \\
MDD-2 & $-9.612(3)$ & $14.4(1)$ \\
B & $-6.521(2)$ & $14.8(1)$ \\
S & $-6.179(2)$ & $34.3(3)$ \\
MFM & $-2.098(1)$ & $25.6(2)$ \\
\hline
\end{tabular}

${ }^{a}$ The figure in parentheses is the estimated error to the last significant figure quoted.

where the scattering amplitude $f(\theta) \equiv f(\theta, k)$ is proportional to the fully on-shell $t$-matrix,

$$
\begin{aligned}
f(\theta) & =-(4 \pi)^{-1} t\left(\mathbf{k}, \mathbf{k}^{\prime} ; k^{2}\right) ; \quad k^{2}=k^{\prime 2} \\
& =(2 i k)^{-1} \sum_{l}(2 l+1)\left(e^{i \delta_{l}(k)}-1\right) P_{l}(\cos \theta)
\end{aligned}
$$

and where $\theta=\cos ^{-1}\left(\hat{k} \cdot \hat{k}^{\prime}\right)$ is the $\mathrm{CM}$ scattering angle. The total cross section is thus simply expressed in terms of the phase shifts as

$$
\begin{aligned}
\sigma_{T}(k) & =2 \pi \int_{0}^{\pi} d \theta \sin \theta(d \sigma / d \Omega) \\
& =4 \pi k^{-2} \sum_{l}(2 l+1)\left[1+(-1)^{l}\right] \sin ^{2} \delta_{l}
\end{aligned}
$$

The total cross section for ${ }^{4} \mathrm{He}-{ }^{4} \mathrm{He}$ scattering obtained for each of the five potentials using Eq. (16) is shown in Fig. 5 for relative momenta $k \leq 4 \AA^{-1}$, which corresponds to velocities $v_{1}=2 \hbar k / m_{4}$ of the projectile on a stationary target $\left(v_{2}=0\right)$ up to about $1300 \mathrm{~m} / \mathrm{sec}$. It was found to be necessary to include partial waves up to $l=18$ to obtain results accurate to better than $\frac{1}{2} \%$ in each case for $k \sim 4 \AA^{-1}$. The typical oscillatory behavior of the cross section as a function of momentum, due to symmetrization of the wave function, is clearly observed.

Corresponding differential cross sections as a function of the $\mathrm{CM}$ seattering angle $\theta$ are shown in Fig. 6 for three different values of the relative momentum, chosen to correspond approximately to the positions of the first three extrema of the total cross sections shown in Fig. 5-the minima corresponding to cases (i) and (iii), and the maximum corresponding to (ii). 


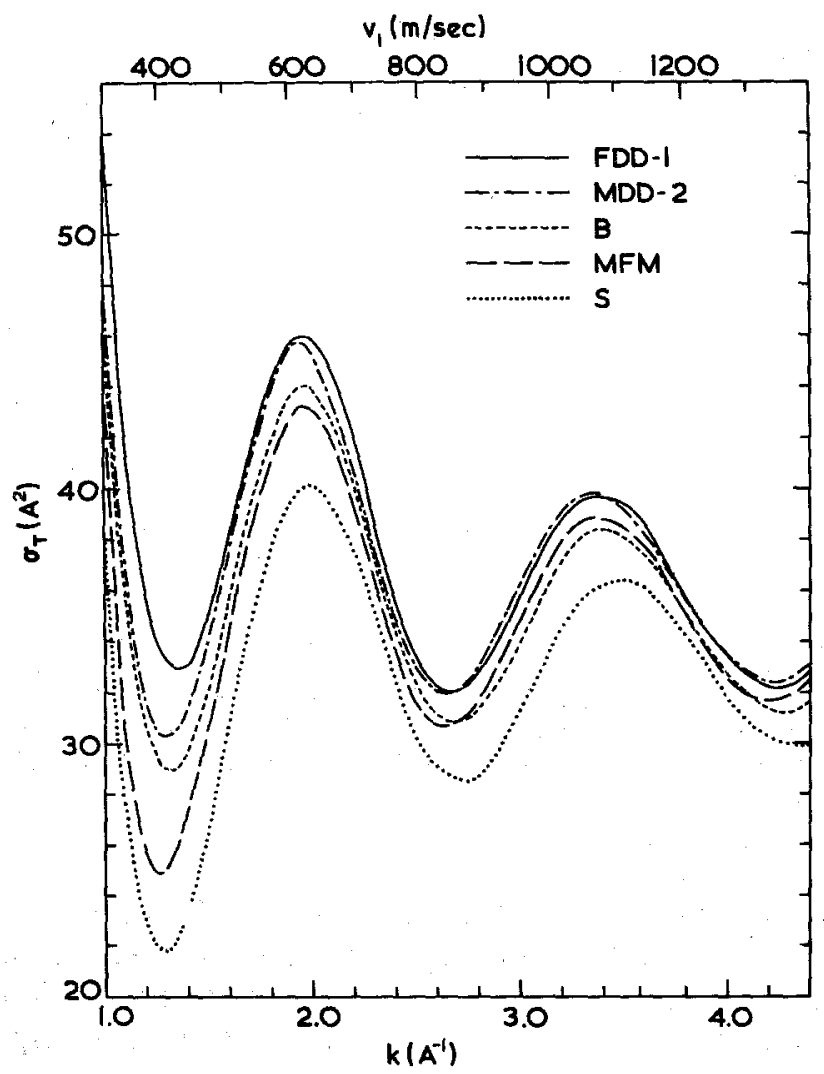

Fig. 5. The total cross section $\sigma_{T}$ for ${ }^{4} \mathrm{He}-{ }^{4} \mathrm{He}$ scattering as a function of the relative momentum $k$ for each of the five potentials considered. The scale $v_{1}=2 \hbar k / m_{4}$ represents the corresponding velocity of a projectile atom on a stationary target atom, $v_{2}=0$.

We have also considered the so-called "viscosity cross section" $\sigma_{\eta}$, which is that particular moment of the differential cross section defined by

$$
\sigma_{n}=2 \pi \int_{0}^{\pi} d \theta \sin ^{3} \theta(d \sigma / d \Omega)
$$

which is instrumental in determining the dilute gas viscosity coefficient. ${ }^{69}$ Use of Eqs. (14) and (15) allows Eq. (17) to be rewritten as (for ${ }^{4} \mathrm{He}-{ }^{4} \mathrm{He}$ )

$$
\sigma_{\eta}=\frac{2 \pi}{k^{2}} \sum_{l} \frac{(l+1)(l+2)}{(l+3 / 2)}\left[1+(-1)^{l}\right] \sin ^{2}\left(\delta_{l+2}-\delta_{l}\right)
$$




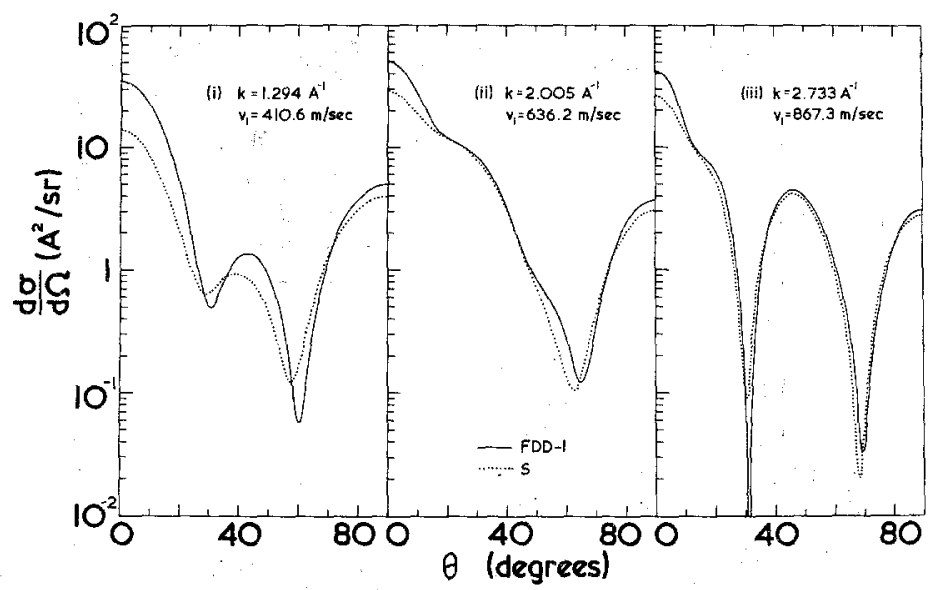

Fig. 6. The differential cross section $d \sigma / d \Omega$ for ${ }^{4} \mathrm{He}-{ }^{4} \mathrm{He}$ scattering as a function of $\mathrm{CM}$ scattering angle $\theta$ at three different relative momenta $k$. Cases (i) and (iii) are for values of $k$ close to those that yield minima in the total cross section, and case (ii) is for a value of $k$ close to the intervening maximum. The results are shown in each case for the FDD-1 potential (solid line) and the $S$ potential (dotted line).

The viscosity cross section for ${ }^{4} \mathrm{He}$ calculated from Eq. (18) is shown in Fig. 7. In both Figs. 6 and 7 we display results only for the two interactions FDD-1 and S. The corresponding curves for the other three potentials are similar and in both cases generally lie between the two curves displayed, as is shown in Fig. 5 for the total cross section.

\section{SUMMARY AND CONCLUSIONS}

In summary, we have carried out a series of computations for a family of semiphenomenological He-He potentials, obtaining binding energies of the possible molecules of the isotopes of $\mathrm{He}$, and low-energy scattering properties for the ${ }^{3} \mathrm{He}-{ }^{3} \mathrm{He}$ and ${ }^{4} \mathrm{He}-{ }^{4} \mathrm{He}$ systems.

For the bound states, we solve a nonlinear (in energy) eigenvalue equation in a momentum representation, obtaining molecular binding energies accurate to better than $10^{-1} \%$. None of the potentials considered is sufficiently attractive to sustain a bound state in the ${ }^{3} \mathrm{He}-{ }^{3} \mathrm{He}$ system. All of the potentials bind the $\left({ }^{6} \mathrm{He}\right)_{2}$ molecule and two of the potentials (FDD-1 and MDD-2) can form a weakly bound $\left({ }^{4} \mathrm{He}\right)_{2}$ molecule. Only the S potential fails to bind the ${ }^{4} \mathrm{He}-{ }^{6} \mathrm{He}$ molecule (see Fig. 2 and Table IV). Our calculations confirm that the $\left({ }^{4} \mathrm{He}\right)_{2}$ molecule is at best by about $50 \mathrm{mK}$ (if at all). 


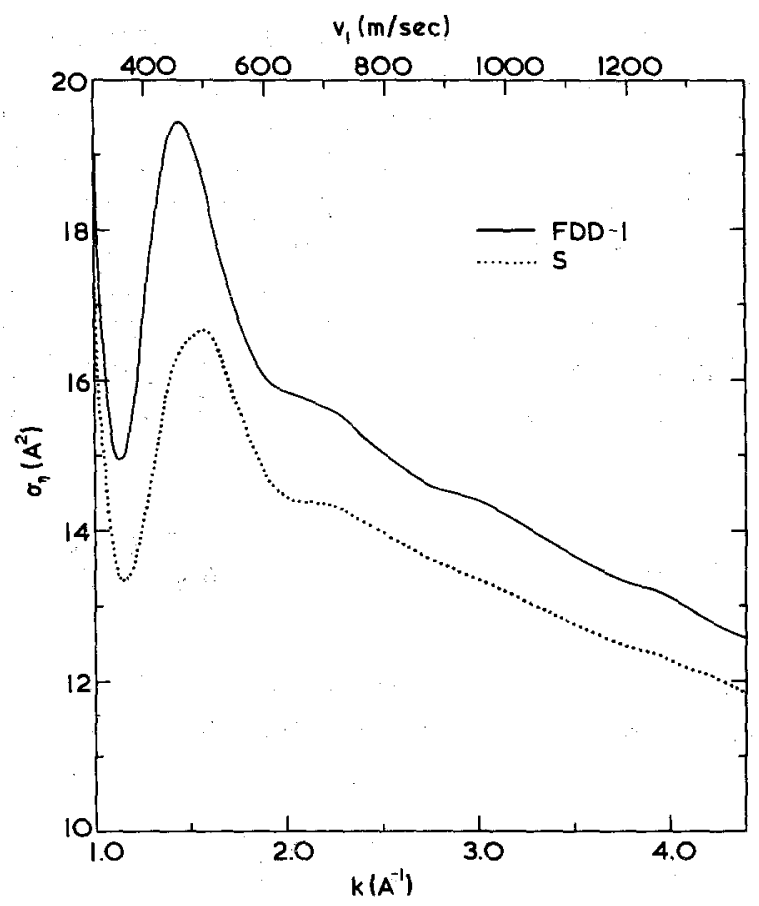

Fig. 7. The viscosity cross section $\sigma_{\eta}$ for ${ }^{4} \mathrm{He}$ as a function of the relative momenta $k$ for the two potentials FDD-1 (solid line) and $\mathrm{S}$ (dotted line).

For low-energy scattering we calculate the fully off-shell $k$-matrix in a momentum representation, employing discrete quadrature techniques to reduce the inhomogeneous integral equation to a finite-dimensional matrix equation. For ${ }^{3} \mathrm{He}$ we compute the first seven partial-wave phase shifts as a function of the relative momentum (see Fig. 3) and summarize the differences in the scattering at low energies between the potentials by an effective range expansion. The scattering length is accurate to better than $10^{-1} \%$ and is quite sensitive to which class of potential is used. In ${ }^{4} \mathrm{He}$, total cross sections and viscosity cross sections are computed as a function of the relative momentum, and differential cross sections are computed at three relative momenta as a function of the CM scattering angle (see Figs. 5-7). The differences between the potentials for any of these data are small and probably not experimentally resolvable.

It should be emphasized that most of the uncertainty in the $\mathrm{He}-\mathrm{He}$ potential occurs in a region about the attractive minimum, and a determination of the binding energy of either of the molecules $\left({ }^{4} \mathrm{He}\right)_{2}$ or ${ }^{4} \mathrm{He}^{-}{ }^{6} \mathrm{He}$ 
would go a long way in resolving this uncertainty. In principle an experimental determination of the ${ }^{4} \mathrm{He}-{ }^{6} \mathrm{He}$ binding energy could be extrapolated to find the $\left({ }^{4} \mathrm{He}\right)_{2}$ binding energy (see Fig. 2). However, atomic ${ }^{6} \mathrm{He}$ is unstable and therefore experiments using it are liable to prove difficult. In general the largest differences in the scattering data among the model potentials occur at low energy, and measurements of low-energy total cross sections would be valuable. Also, very low-temperature measurements of the dilute gas viscosity could in principle serve as an alternative method of obtaining the same information. This can be understood by the following simple argument. At low temperatures the viscosity coefficient $\eta$ for a Bose system $\left({ }^{4} \mathrm{He}\right)$ is given by $^{69}$

$$
\eta^{-1} \propto\left(\frac{2 m_{4} k_{B} T}{\hbar^{2}}\right)^{-1 / 2} \int_{0}^{\infty} d x x^{7} \exp \left(-x^{2}\right) \sigma_{\eta}\left[\left(\frac{2 m_{4} k_{B} T}{\hbar^{2}}\right)^{1 / 2} x\right]
$$

where $\sigma_{\eta}$ is given by Eq. (18). In the low-temperature limit only small values of the relative momenta $x$ are important, and from Eq. (18) we find,

$$
\sigma_{\eta}(k) \underset{k \rightarrow 0}{\longrightarrow} 16 \pi a_{0}^{2} / 3
$$

where $a_{0}$ is the $S$-wave scattering length of Eq. (13). This expression will be a good approximation whenever $k a_{0} \ll 1$. From the above integral expression it is clear that contributions to $\eta^{-1}$ become small for $x^{2} \gg 7 / 2$, which in turn implies

$$
\left(\frac{2 m_{4} k_{B} T}{\hbar^{2}}\right)^{1 / 2} \ll \frac{(2 / 7)^{1 / 2}}{a_{0}}
$$

If these conditions are satisfied, which in ${ }^{4} \mathrm{He}$ would take place at temperatures below $300 \mathrm{mK}$, then $\eta^{-1} \propto a_{0}^{2}$. Thus, if even fairly rough viscosity measurements could be performed in this region, they could greatly help to distinguish the potentials used here since these show a wide variation for the parameter $a_{0}$.

\section{ACKNOWLEDGMENTS}

Useful discussions with Drs. K. Kulander and J. M. Irvine are gratefully acknowledged. One of us (H.B.G.) is grateful to Prof. F. Torello for an enlightening communication. 


\section{REFERENCES}

1. William E. Keller, Helium-3 and Helium-4 (Plenum Press, New York, 1969).

2. H. A. Bethe and E. E. Salpeter, in Handbuch der Physik, S. Flügge, ed. (Springer, Berlin, 1957), Vol. 35, Part 1, p. 252.

3. J. O. Hirschfelder, J. Chem. Phys. 43, S199 (1965).

4. J. de Boer and A. Michels, Physica 5, 945 (1939); 6, 409 (1939).

5. R. Peierls, Quantum Theory of Solids (Clarendon Press, Oxford, 1955); T. R. Koehler, Phys. Rev. 144, 789 (1966); 165, 942 (1968).

6. D. Pines and P. Nozieres, The Theory of Quantum Liquids, Vol. I (Benjamin, New York, 1966).

7. W. E. Keller, Phys. Rev. 97, 1 (1955); 98, 1571 (1955); 100, 1021, 1790 (1955).

8. K. Fokkens, W. Vermeer, K. W. Taconis, and R. de Bruyn Ouboter, Physica 30, 2153 (1964).

9. P. J. Bendt, Phys. Rev. 110, 85 (1958).

10. E. W. Becker and R. Misenta, Z. Physik 140, 535 (1955).

11. P. Cantini, M. Cavallini, M. G. Dondi, and G. Scoles, Phys. Lett. 27A, 284 (1968).

12. M. G. Dondi, G. Scoles, F. Torello, and H. Pauly, J. Chem. Phys. 51, 392 (1969).

13. M. Cavallini, L. Meneghetti, G. Scoles, and M. Yealland, Phys. Rev. Lett. 24, 1469 (1970).

14. P. E. Siska, J. M. Parson, T. P. Schafer, and Y. T. Lee, J. Chem. Phys. 55, 5762 (1971).

15. J. M. Farrar and Y. T. Lee, J. Chem. Phys. 56, 5801 (1972).

16. P. Cantini, M. G. Dondi, G. Scoles, and F. Torello, J. Chem. Phys. 56, 1946 (1972).

17. H. G. Bennewitz, H. Busse, H. D. Dohmann, D. E. Oates, and W. Schrader, Z. Physik 253, 435 (1972)

18. H. G. Bennewitz, H. Busse, H. D. Dohmann, D. E. Oates, and W. Schrader, Phys. Rev. Lett. 29, 533 (1972).

19. W. Aufm Kampe, D. E. Oates, W. Schrader, and H. G. Bennewitz, Chem. Phys. Lett. 18, 323 (1973).

20. R. Feltgen, H. Pauly, F. Torello, and H. Vehmeyer, Phys. Rev. Lett. 30, 820, 1098 (1973).

21. R. Feltgen, K. A. Köhler, H. Pauly, F. Torello, and H. Vehmeyer, in Proc. IV Int. Symposium on Molecular Beams, Cannes, 1973.

22. A. L. J. Burgmans, J. M. Farrar, and Y. T. Lee, J. Chem. Phys, 64, 1345 (1976).

23. J. E. Jordan and I. Amdur, J. Chem. Phys. 46, 165 (1967).

24. U. Buck, Rev. Mod. Phys. 42, 369 (1974).

25. J. L. Yntema and W. G. Schneider, J. Chem. Phys. 18, 646 (1950).

26. E. A. Mason and W. E. Rice, J. Chem. Phys. 22, 522 (1954).

27. R. B. Bernstein and F. A. Morse, J. Chem. Phys. 40, 917 (1964).

28. J. N. Murrel and G. Shaw, Mol. Phys. 15, 325 (1968).

29. A. A. Frost and B. Musulin, J. Chem. Phys. 22, 1017 (1954).

30. L. W. Bruch and I. J. McGee, J. Chem. Phys. 46, 2959 (1967); 52, 5884 (1970).

31. D. E. Beck, Mol. Phys. 14; 311 (1968); 15, 322 (1968).

32. G. Sposito, Phys. Rev. A 2, 948 (1970); J. Low Temp. Phys. 3, 491 (1970).

33. K. M. Howell, Proc. Phys. Soc. (London) A 67, 705 (1954).

34. P. R. Fontana, Phys. Rev. 123, 1865 (1961).

35. Y. M. Chan and A. Dalgarno, Proc. Phys. Soc. (London) 86, 777 (1965).

36. R. J. Bell, Proc. Phys. Soc. (London) 86, 17 (1965).

37. W. D. Davison, Proc. Phys. Soc. (London) 87, 133 (1966).

38. N. R. Kestner, J. Chem. Phys. 45, 208 (1966).

39. A. Dalgarno and G. Victor, Proc. Phys. Soc. (London) 90, 605 (1967).

40. K. T. Tang and M. Karplus, Phys. Rev. 171, 70 (1968).

41. R. G. Gordon, J. Chem. Phys. 48, 3929 (1968).

42. D. A. McQuarrie, J. Terebey, and S. J. Shire, J. Chem. Phys. 51, 4683 (1969).

43. P. E. Phillipson, Phys. Rev. 125, 1981 (1962).

44. T. L. Gilbert and A. C. Wahl, J. Chem. Phys. 47, 3425 (1967).

45. G. H. Matsumoto, C. F. Bender, and E. R. Davidson, I. Chem. Phys. 46, 402 (1967). 
46. D. J. Klein, C. E. Rodriguez, J. C. Browne, and F. A. Matsen, J. Chem. Phys. 47, 4862 (1967).

47. H. F. Schaefer, D. R. McLaughlin, F. E. Harris, and B. J. Alder, Phys. Rev. Lett. 25,988 (1970).

48. P. J. Bertoncini and A. C. Wahl, Phys. Rev. Lett. 25, 991 (1970); J. Chem. Phys. 58, 1680 (1973).

49. D. R. McLaughlin and H. F. Schaefer, Chem. Phys. Lett. 12, 244 (1971).

50. B. Liu and A. D. McLean, J. Chem. Phys. 59, 4557 (1973).

51. W. L. Taylor and J. M. Keller, J. Chem. Phys. 54, 647 (1971).

52. E. Østgaard, Phys. Rev. 170, 257 (1968); 171, 248 (1968); 176, 351 (1968); 180, 263 (1969); 187, 371 (1969).

53. H. B. Ghassib, Ph.D. Thesis, University of Manchester (July 1974), unpublished; H. B. Ghassib, R. H. Ibarra, and J. M. Irvine, Ann. Phys. (NY) 85, 378 (1974); H. B. Ghassib and J. M. Irvine, J. Low Temp. Phys, 18, 201 (1975).

54. R. D. Murphy and R. O. Watts, J. Low Temp. Phys. 2, 507 (1970).

55. R. D. Murphy, Phys. Rev. A 5, 331 (1972).

56. A. G. Gibbs and O. K. Harling, Phys. Lett. 36A, 203 (1971).

57. H. R. Glyde, Can. J. Phys. 49, 761 (1971).

58. J. P. Hansen, Phys. Lett. 34A, 25 (1971)

59. R. D. Etters, J. C. Raich, and C. Cochran, J. Low Temp. Phys. 9, 53 (1972).

60. E. K. Achter and L. Meyer, Phys. Rev. 188, 291 (1969).

61. O. Sinanoglu, in Intermolecular Forces, J O. Hirschfelder, ed. (Wiley, New York, 1967), p. 283.

62. K. A. Brueckner and J. L. Gammel, Phys. Rev. 109, 1040 (1958).

63. D. E. Beck, J. Chem. Phys. 50, 541 (1969).

64. A. Pais and G. E: Uhlenbeck, Phys. Rev. 116, 250 (1959).

65. J. de Boer, J. Van Kranendonk, and K. Compaan, Physica 16, 545 (1950).

66. J. E. Kilpatrick, W. E. Keller, E. F. Hammel, and N. Metropolis, Phys, Rev. 94, 1103 (1954).

67. N. Levinson, Kgl. Danske Videnskab Selskab, Mat.-Fys. Medd. 25, No. 9 (1949).

68. L. I. Schiff, Quantum Mechanics, 3rd ed. (McGraw-Hill, New York, 1968), p. 370.

69. N. F. Mott and H. S. W. Massey, The Theory of Atomic Collisions, 3rd ed. (Oxford University Press, Oxford, 1965), Chapter XIX.

70. J. H. E. Mattauch, W. Thiele, and A. H. Wapstra, Nucl. Phys. 67, 1 (1965).

71. E. R. Cohen and B. N. Taylor, J. Phys. Chem. Ref. Data 2, 663 (1973). 